\title{
Clear cell meningiomas are defined by a highly distinct DNA methylation profile and mutations in SMARCE1
}

\author{
Philipp Sievers ${ }^{1,2}$ - Martin Sill ${ }^{3,4} \cdot$ Christina Blume $^{1,2,5} \cdot$ Arnault Tauziede-Espariat $^{6} \cdot$ Daniel Schrimpf $^{1,2}$. \\ Damian Stichel ${ }^{1,2}$. David E. Reuss ${ }^{1,2} \cdot$ Helin Dogan $^{1,2}$. Christian Hartmann ${ }^{7}$. Christian Mawrin ${ }^{8} \cdot$ Martin Hasselblatt $^{9}$. \\ Walter Stummer ${ }^{10}$. Uta Schick ${ }^{11}$. Jürgen Hench ${ }^{12}$. Stephan Frank ${ }^{12}$. Ralf Ketter ${ }^{13}$. Leonille Schweizer ${ }^{14,15}$. \\ Jens Schittenhelm ${ }^{16}$. Stéphanie Puget ${ }^{17}$. Sebastian Brandner ${ }^{18,19} \cdot$ Zane Jaunmuktane $^{18,20}$ • Benno Küsters ${ }^{21}$. \\ Zied Abdullaev ${ }^{22}$ - Melike Pekmezci' ${ }^{23}$. Matija Snuderl ${ }^{24}$ - Miriam Ratliff ${ }^{25}$. Christel Herold-Mende ${ }^{26}$. \\ Andreas Unterberg ${ }^{27} \cdot$ Kenneth Aldape ${ }^{22} \cdot$ David W. Ellison $^{28} \cdot$ Pieter Wesseling $^{29,30} \cdot$ Guido Reifenberger $^{31,32}$. \\ Wolfgang Wick ${ }^{33,34}$. Arie Perry ${ }^{23,35}$. Pascale Varlet ${ }^{6}$. Stefan M. Pfister ${ }^{3,4,36} \cdot$ David T. W. Jones $^{3,37}$. \\ Andreas von Deimling ${ }^{1,2} \cdot$ Felix Sahm ${ }^{1,2,3}$ (1) The German Consortium “Aggressive Meningiomas"
}

Received: 19 October 2020 / Revised: 16 November 2020 / Accepted: 19 November 2020 / Published online: 14 December 2020 (c) The Author(s) 2020

\begin{abstract}
Clear cell meningioma represents an uncommon variant of meningioma that typically affects children and young adults. Although an enrichment of loss-of-function mutations in the SMARCE1 gene has been reported for this subtype, comprehensive molecular investigations are lacking. Here we describe a molecularly distinct subset of tumors $(n=31)$, initially identified through genome-wide DNA methylation screening among a cohort of 3093 meningiomas, of which most were diagnosed histologically as clear cell meningioma. This cohort was further supplemented by an additional 11 histologically diagnosed clear cell meningiomas for analysis $(n=42)$. Targeted DNA sequencing revealed SMARCE1 mutations in 33/34 analyzed samples, accompanied by a nuclear loss of expression determined via immunohistochemistry and a decreased SMARCE1 transcript expression in the tumor cells. Analysis of time to progression or recurrence of patients within the clear cell meningioma group $(n=14)$ in comparison to those with meningioma WHO grade $2(n=220)$ revealed a similar outcome and support the assignment of WHO grade 2 to these tumors. Our findings indicate the existence of a highly distinct epigenetic signature of clear cell meningiomas, separate from all other variants of meningiomas, with recurrent mutations in the SMARCE1 gene. This suggests that these tumors may arise from a different precursor cell population than the broad spectrum of the other meningioma subtypes.
\end{abstract}

Keywords Brain tumor $\cdot$ Meningioma $\cdot$ Clear cell $\cdot S M A R C E 1 \cdot$ DNA methylation profile

\section{Introduction}

Meningioma is the most common primary central nervous system (CNS) neoplasm, accounting for about a third of all brain tumors $[10,16]$. Clear cell meningioma represents an

Philipp Sievers and Martin Sill have shared authorship.

Supplementary Information The online version contains supplementary material available at https://doi.org/10.1007/s0040 1-020-02247-2.

Felix Sahm

felix.sahm@med.uni-heidelberg.de

Extended author information available on the last page of the article uncommon variant of meningioma that typically affects children and young adults [12, 21-23, 26]. Histologically, it is characterized by sheets of rounded or polygonal clear cells and perivascular and interstitial collagen [10]. Clear cell meningioma is associated with a more aggressive behavior [29] and is, therefore, classified as a World Health Organization (WHO) grade 2 tumor [10]. Genetically, an enrichment of loss-of-function mutations in the SMARCE1 gene encoding a subunit of the SWI/SNF chromatin remodeling complex has been reported for this meningioma subtype [22, 23]. NF2 mutations, common in other meningiomas, are rare in this subtype [21]. Yet, it remains elusive whether clear cell meningioma is merely a morphological variant (albeit with prognostic implications) that can in principle be associated 
with various driving alterations, or whether the predominance of SMARCE1 alterations rather points to a defining set of molecular underpinnings unique to these tumors.

Investigating the DNA methylation landscape of meningiomas, we identified a cluster of 31 tumor samples in a cohort of 3093 meningiomas $(\sim 1 \%)$ that formed a highly distinct group, well detached from the other meningiomas, of which most cases were diagnosed histologically as clear cell meningioma. Based on this observation we collected additional morphologically identified clear cell meningiomas $(n=11)$ and performed further molecular workup on the whole cohort $(n=42)$ using DNA methylation profiling, targeted next-generation DNA sequencing and RNA sequencing.

\section{Materials and methods}

\section{Sample collection}

Tumor samples and retrospective clinical data from 42 patients were provided by multiple national and international collaborating centers and collected at the Department of Neuropathology of the University Hospital Heidelberg (Germany). Case selection was based on unsupervised hierarchical clustering of genome-wide DNA methylation data in a cohort of 3,093 meningiomas that revealed a molecularly distinct group of tumors comprising 31 samples, of which most were diagnosed histologically as clear cell meningioma. Additionally, 11 histologically diagnosed clear cell meningiomas were subsequently integrated into analyses to verify that the specific cluster is made up by this subtype of meningioma. Analysis of tissue and clinical data was performed in accordance with local ethics regulations. Clinical details of the patients are listed in Supplementary Table 1 (online resource).

\section{Immunohistochemistry}

Immunohistochemistry was performed on a Ventana BenchMark ULTRA Immunostainer (Ventana Medical Systems, Tucson, AZ, USA) for all cases with sufficient tissue (for EMA and SSTR2A $n=22$; for SMARCE1 $n=25$; for Ki-67 $n=26)$. Antibodies were directed against: epithelial membrane antigen (EMA; Clone GP1.4, mouse monoclonal, dilution 1:1000, Thermo Fisher Scientific, Fremont, CA, USA), somatostatin receptor 2A (SSTR2A; SS-8000-RM, rabbit monoclonal, dilution 1:10, Biotrend, Cologne, Germany), Ki-67 (clone MIB-1, mouse monoclonal, 1:100 dilution, Dako Agilent, Santa Clara, CA, USA) and SMARCE1 (HPA003916, rabbit polyclonal, dilution 1:700, SigmaAldrich, St. Louis, MO, USA).
DNA methylation array processing and copy number profiling

Genomic DNA was extracted from formalin-fixed and paraffin-embedded (FFPE) tissue samples. DNA methylation profiling of all samples was performed using the Infinium MethylationEPIC (850k) BeadChip (Illumina, San Diego, CA, USA) or Infinium HumanMethylation450 (450k) BeadChip array (Illumina) as previously described [2] All computational analyses were performed in $\mathrm{R}$ version 3.3.1 (R Development Core Team, 2016; https://www.Rproject.org). Copy-number variation analysis from $450 \mathrm{k}$ and EPIC methylation array data was performed using the conumee Bioconductor package version 1.12.0. Raw signal intensities were obtained from IDAT-files using the minfi Bioconductor package version 1.21.4 [1]. Illumina EPIC samples and 450k samples were merged to a combined data set by selecting the intersection of probes present on both arrays (combineArrays function, minfi). Each sample was individually normalized by performing a background correction (shifting of the $5 \%$ percentile of negative control probe intensities to 0 ) and a dye-bias correction (scaling of the mean of normalization control probe intensities to 10,000 ) for both color channels. Subsequently, a correction for the array type (450k/EPIC) was performed by fitting univariable, linear models to the log2-transformed intensity values (removeBatchEffect function, limma package version 3.30.11). The methylated and unmethylated signals were corrected individually. Beta-values were calculated from the retransformed intensities using an offset of 100 (as recommended by Illumina). All samples were checked for duplicates by pairwise correlation of the genotyping probes on the $450 \mathrm{k} / 850 \mathrm{k}$ array. To perform unsupervised non-linear dimension reduction, the remaining probes after standard filtering [2] were used to calculate the 1-variance weighted Pearson correlation between samples. The resulting distance matrix was used as input for t-SNE analysis ( $t$-distributed stochastic neighbor embedding; Rtsne package version 0.13). The following non-default parameters were applied: theta $=0, \mathrm{pca}=\mathrm{F}$, max_iter $=15,000$ perplexity $=20$.

\section{Targeted next-generation DNA sequencing and mutational analysis}

Genomic DNA was extracted from FFPE tumor tissue samples of 34 patients within the cohort using the automated Maxwell system with the Maxwell 16 FFPE Plus LEV DNA Purification Kit (Promega, Madison, WI, USA), according to the manufacturer's instructions. Matched normal DNA was extracted from blood samples 
of four of the affected individuals using the Maxwell 16 Blood DNA Purification Kit (Promega). Capture-based next-generation DNA sequencing was performed on a NextSeq 500 instrument (Illumina) as previously described [18] using a custom brain tumor panel covering the entire coding and selected intronic and promoter regions of 130 genes of particular relevance in central nervous system tumors. Reads were aligned against the reference genome (GRch37).

\section{RNA sequencing and analysis}

RNA was extracted from FFPE tissue samples using the automated Maxwell system with the Maxwell 16 LEV RNA FFPE Kit (Promega, Madison, WI, USA), according to the manufacturer's instructions. Transcriptome analysis using messenger RNA (mRNA) sequencing of samples for which RNA of sufficient quality and quantity was available (clear cell meningioma $(n=15), N F 2$-mutant meningioma $(n=12))$ was performed on a NextSeq 500 (Illumina) as previously described [24]. Fastq files from transcriptome sequencing were used for de novo annotation of fusion transcripts using the defuse [14] and arriba (https://github.com/suhrig/arriba/) algorithms with standard parameters. Alignment to the human genome (GRCh37) was performed with the STAR aligner [4] and reads were then quantified using RSEM [9]. All further analysis was performed in R (version 3.6.0; R Core Team, 2019) using the DESeq2 package (version 1.26.0) [11]. Principal Component Analysis (PCA) was performed after variance stabilizing transformation of the count data and normalization with respect to library size. Similarities between samples were determined by computing Manhattan distances on the variance stabilized data followed by unsupervised hierarchical clustering. Differential expression testing was performed on raw count data after fitting a negative binomial model. $P$-values were adjusted for multiplicity by applying the Benjamini-Hochberg correction.

\section{Statistical analysis}

Statistical analysis was performed using GraphPad Prism 8 (GraphPad Software, La Jolla, CA, USA). Data on survival could be retrospectively retrieved for 14 patients. Distribution of time to progression or recurrence (TTP) after surgery was estimated by the Kaplan-Meier method and compared between groups with the log-rank test. $P$-values below 0.05 were considered significant.

\section{Results}

\section{DNA methylation profiling reveals a highly distinct epigenetic signature of clear cell meningioma}

Investigating the DNA methylation landscape of meningiomas, we identified a group of 31 tumor samples in a cohort of 3093 meningiomas that formed a highly distinct cluster, well detached from the other meningiomas (Suppl. Figure 1, online resource). Intriguingly, most (18/31) cases in this initial cluster had been diagnosed histologically as clear cell meningioma, followed by atypical meningioma as the second most common diagnosis (Suppl. Table 1, online resource). Based on this observation we collected additional clear cell meningiomas based on their morphology $(n=11)$ that subsequently also all grouped with this specific cluster by DNA methylation profiling. A more focused t-distributed stochastic neighbor embedding (t-SNE) analysis of DNA methylation patterns confirmed the distinct nature of this group (Fig. 1). In contrast, none of the other meningioma subtypes with characteristic alterations, e.g. mutations in $N F 2, T R A F 7 / K L F 4$ or BAP1, or YAP1 fusion seem to form

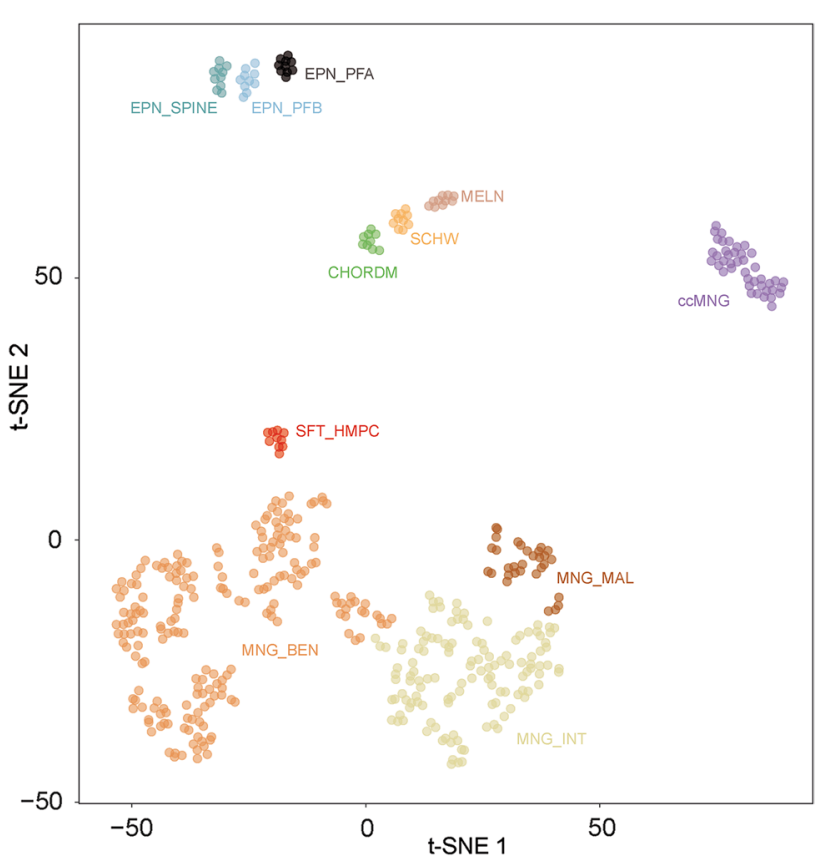

Fig. 1 t-distributed stochastic neighbor embedding (t-SNE) analysis of DNA methylation profiles of the 42 clear cell meningiomas (ccMNG) alongside selected reference samples. Reference DNA methylation classes: meningioma benign (MNG_BEN), meningioma intermediate (MNG_INT), meningioma malignant (MNG_MAL), ependymoma posterior fossa group A (EPN_PFA), ependymoma posterior fossa group B (EPN_PFB), ependymoma spinal (EPN_SPINE), solitary fibrous tumor/hemangiopericytoma (SFT_HMPC), schwannoma (SCHW), chordoma (CHORD) and secondary/metastatic melanoma (MELN) 
independent epigenetic clusters (Suppl. Figure 2, online resource). Analysis of copy number profiles derived from DNA methylation data revealed recurrent chromosomal aberrations within the clear cell meningioma group including chromosome 17q (segmental) loss, chromosome 6q loss and chromosome $22 q$ loss in approximately one-third of the cases (Suppl. Figure 3, online resource).

\section{Clear cell meningiomas are characterized by mutations in SMARCE1}

We next used targeted next-generation sequencing to gain insight into the mutational landscape of the meningiomas in this cluster and identified mutations in SMARCE1 in 33 of the 34 cases $(97 \%)$ with sufficient material available (Fig. 2 and Suppl. Table 1, online resource). Of the all in all 36 detected SMARCE1 mutations (three cases harbored two separate mutations), 32 were nonsense or frameshift mutations predicted to result in complete loss of the protein product (Fig. 2 and Suppl. Table 1, online resource). Immunohistochemical detection of SMARCE1 showed widespread nuclear loss of expression in the tumor cells in all analyzed cases $(n=25)$. The mutant allele frequency for the SMARCE1 mutations was highly variable (mean $58 \%$; range 30-90\%), suggesting a possible biallelic loss of the gene at least in some cases. In four of the cases with matched normal DNA available, the SMARCE1 mutation was confirmed in the germline. Beside SMARCE1, no additional pathogenic mutations were detected (particularly none in the genes implicated in meningioma biology, including NF2, AKT1, KLF4, TRAF7, SMO, SUFU, PTCH1, BAP1, SMARCB1, PTEN, PIK3CA or TERT promoter mutations). In addition, screening of 705 meningioma samples with available sequencing and DNA methylation data revealed
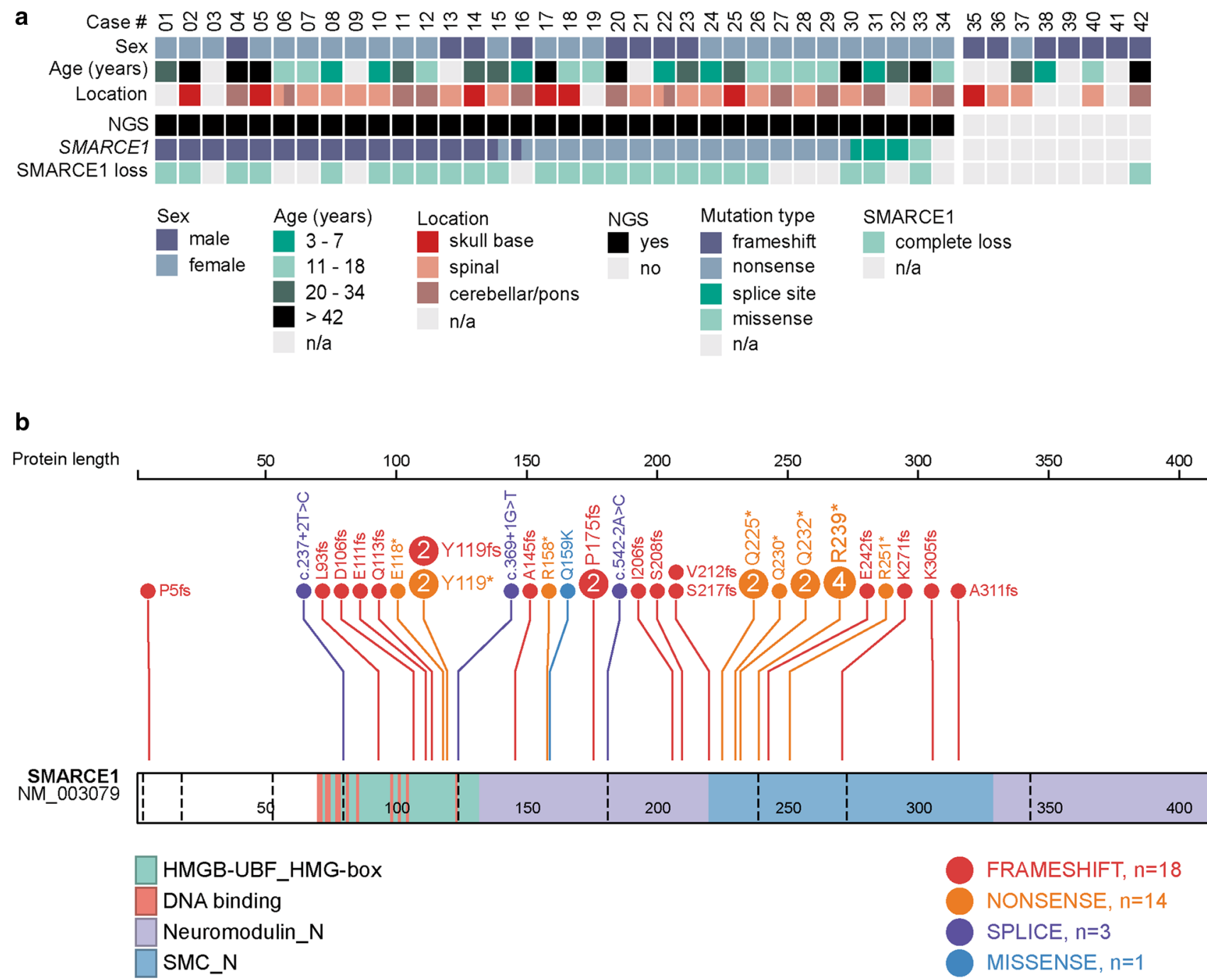

Fig. 2 Clinicopathological characteristics and recurrent genetic alterations of the 42 clear cell meningiomas (a). Visualization of the SMARCE1 mutation profile in the investigated cohort was created using the online tool ProteinPaint available at https://proteinpaint.stjude.org/ (b) 
that SMARCE1 mutations occurred exclusively within the specific epigenetic class of clear cell meningiomas.

\section{Differential gene expression segregates clear cell meningiomas and NF2-mutant meningiomas}

We next performed mRNA sequencing of 15 tumor samples within the specific clear cell cluster and 12 NF2-mutant meningioma samples for comparison. Gene expression variability within the cohort did not correlate with clinical parameters or anatomical location. Unsupervised hierarchical clustering (Fig. 3a) and PCA analysis (Fig. 3b) demonstrated a clear segregation of tumor samples by DNA methylation profile and histology. In addition, quantification of mRNA expression confirmed decreased SMARCE1 expression in tumors within the clear cell cohort as compared to $N F 2$-altered meningiomas (adjusted $p=1.34 \mathrm{e}-11$; Fig. 3c). NF2 transcript levels were downregulated in NF2-mutant meningioma samples, respectively (adjusted $p=1.10 \mathrm{e}-$ 14; Fig. 3d). Interestingly, analysis of EZH2, the catalytic a

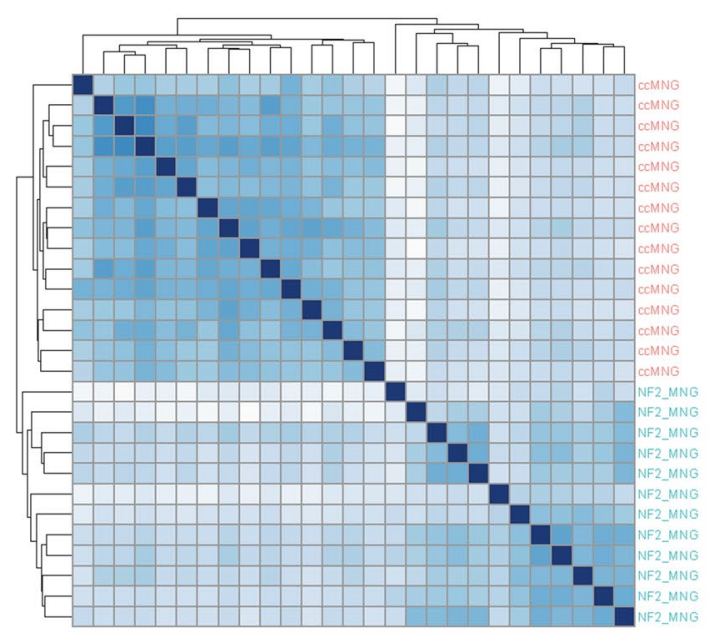

C

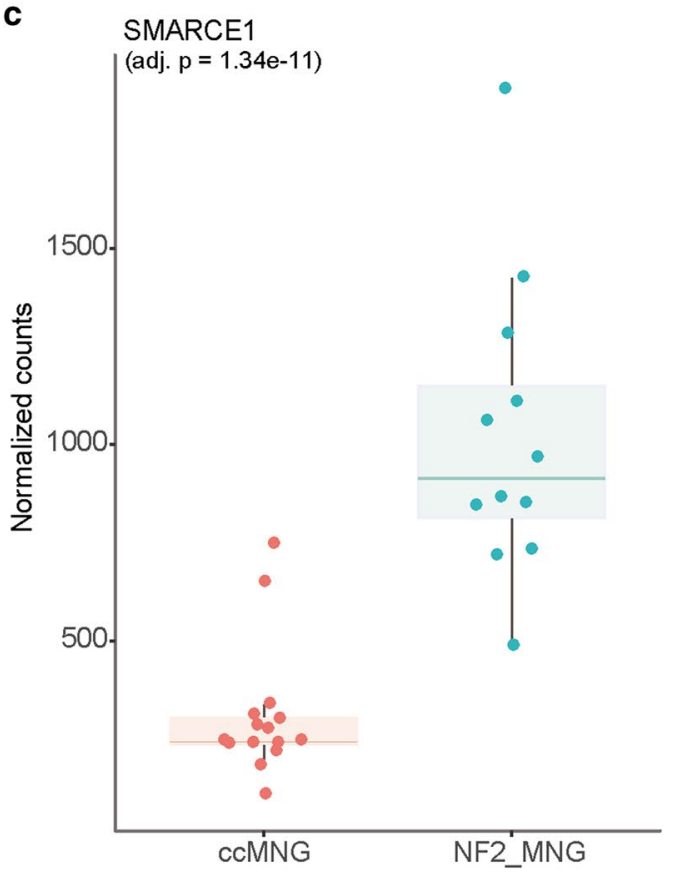

Fig. 3 Differences in gene expression profiles between clear cell meningiomas and NF2-mutant meningiomas. Normalized transcript counts from clear cell meningioma and NF2-mutant meningioma samples clustered by Pearson's correlation coefficient (a) and princi-

b

d
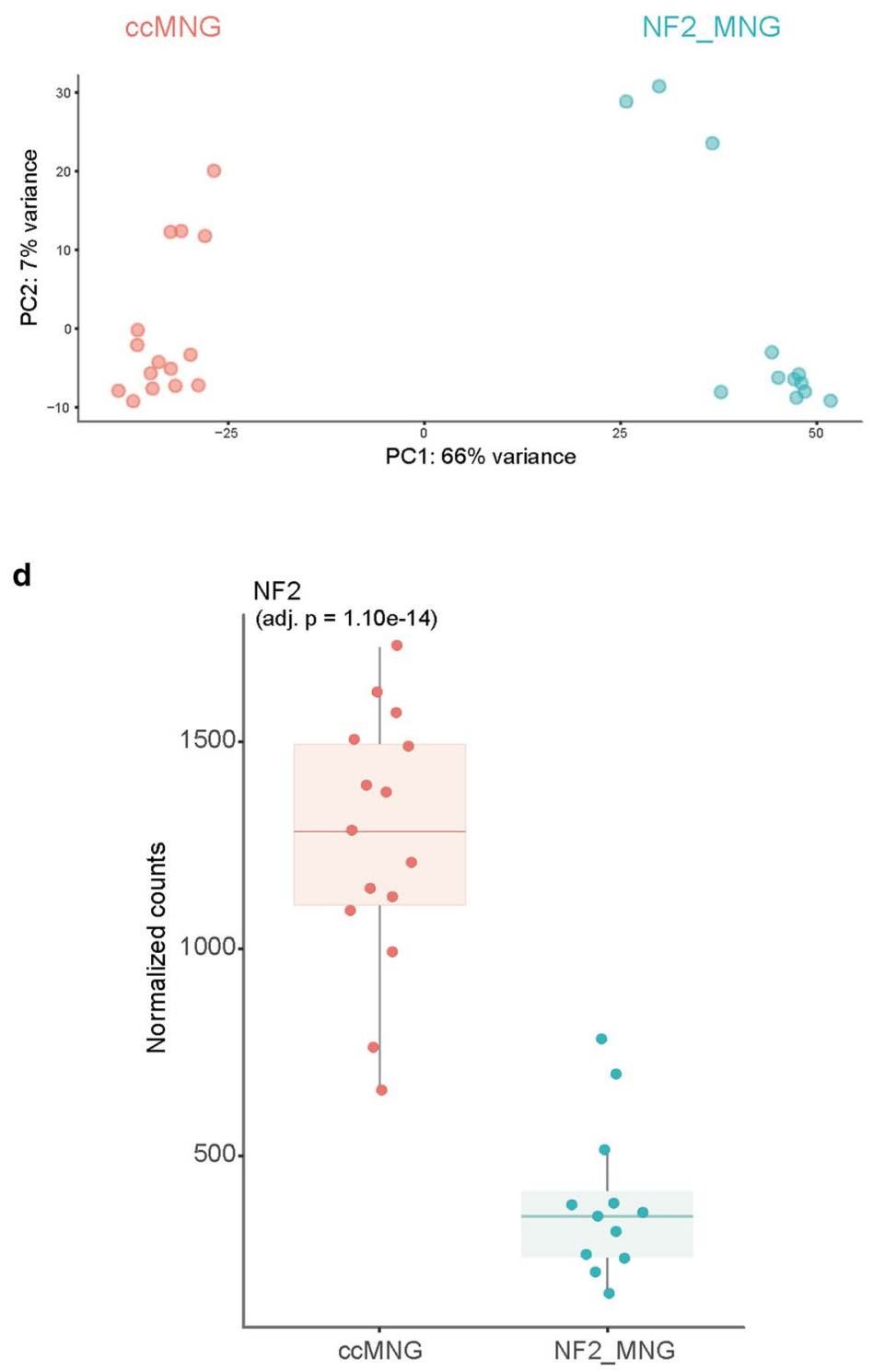

pal component analysis (b). SMARCE1 (c) and NF2 (d) expression in clear cell meningiomas $(n=15)$ determined by RNA-sequencing compared to NF2-mutant meningioma samples $(n=12)$ 
subunit of the PRC2 complex which acts to antagonize the SWI/SNF complex, indicated an increased expression in clear cell meningiomas compared to meningiomas with $N F 2$ mutation (adjusted $p=1.70 \mathrm{e}-07$; Suppl. Figure 4, online resource). Further differentially expressed genes between clear cell meningiomas and $N F 2$-altered meningiomas are highlighted in supplementary Fig. 5 (online resource).

\section{Clinical characteristics and morphological features within the molecularly defined clear cell meningioma cohort}

The majority of the tumors in this molecular group $(n=29 / 42)$ were histologically diagnosed as clear cell meningioma (Suppl. Figure 1, online resource). Histopathological review according to the WHO 2016 classification of tumors of the central nervous system for all cases with sufficient material confirmed these findings. All these tumors histologically showed clearly recognizable cellular areas composed of round to polygonal clear cells with perivascular and interstitial collagen corresponding to the typical histological features of clear cell meningioma
(Fig. 4). All tumors exhibited immunohistochemical expression of EMA and SSTR2A (Fig. 4 and Suppl. Figure 1 , online resource). Mitotic counts were generally low to moderate (between 0 and 1.7 mitosis per $\mathrm{mm}^{2}$ ). Two tumors exhibited a higher count of up to 2.1 and 2.9 mitoses per $\mathrm{mm}^{2}$. The Ki-67 labeling index was very low (1-4\%) in eight tumors, while 14 tumors had an elevated proliferation index of $5-10 \%$ and four of $15-20 \%$. Consistent with previous reports [23], clear cell meningiomas in our series were located in the spine $(n=17)$ as well as intracranially $(n=18)$. Median age at presentation in our cohort was 25 years (range 3-75) with a female predominance (F:M ratio 1.8:1). Outcome data were available for 14 patients within the cohort. In comparison to a cohort of 458 meningiomas of other subtypes (WHO grade 1 and 2) the prediction of outcome seems to be consistent with the current WHO grade 2 assignment: Time to recurrence in patients with clear cell meningiomas in our cohort was significantly worse than in patients with WHO grade 1 meningiomas ( $p=0.003$; Fig. 5a) and not different from that in patients with other meningiomas of WHO grade 2 $(p=0.63$; Fig. 5b).
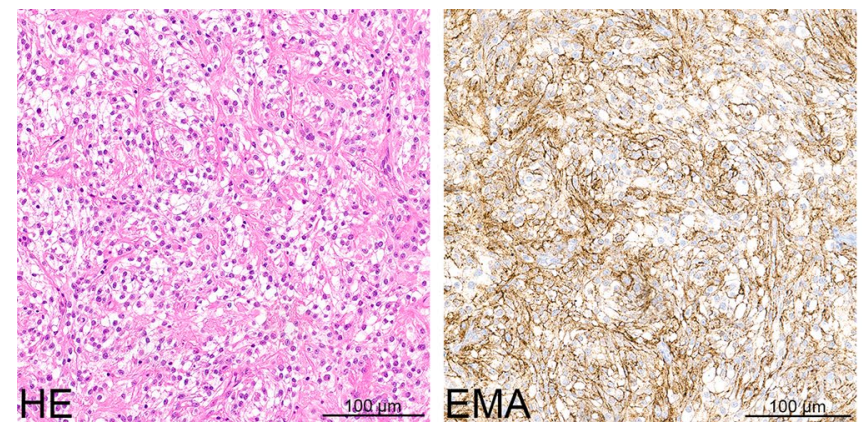

Fig. 4 Morphological and immunohistochemical features of clear cell meningiomas within the cohort. H\&E staining of one of the clear cell meningiomas included in the investigated cohort showing a high
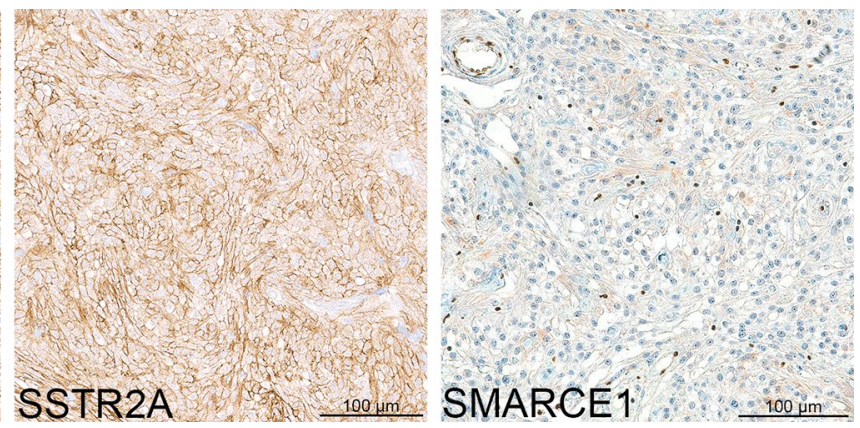

cellularity of round to polygonal clear cells with perivascular and interstitial collagen. Immunohistochemical expression of EMA and SSTR2A as well as nuclear loss of SMARCE1 in the tumor cells
Fig. 5 Time to progression or recurrence (TTP) of 14 patients from the investigated cohort (ccMNG) for whom follow-up data were available compared to TTP of 238 patients with meningioma WHO grade 1 (a) and 220 patients with meningioma WHO grade 2 (b)
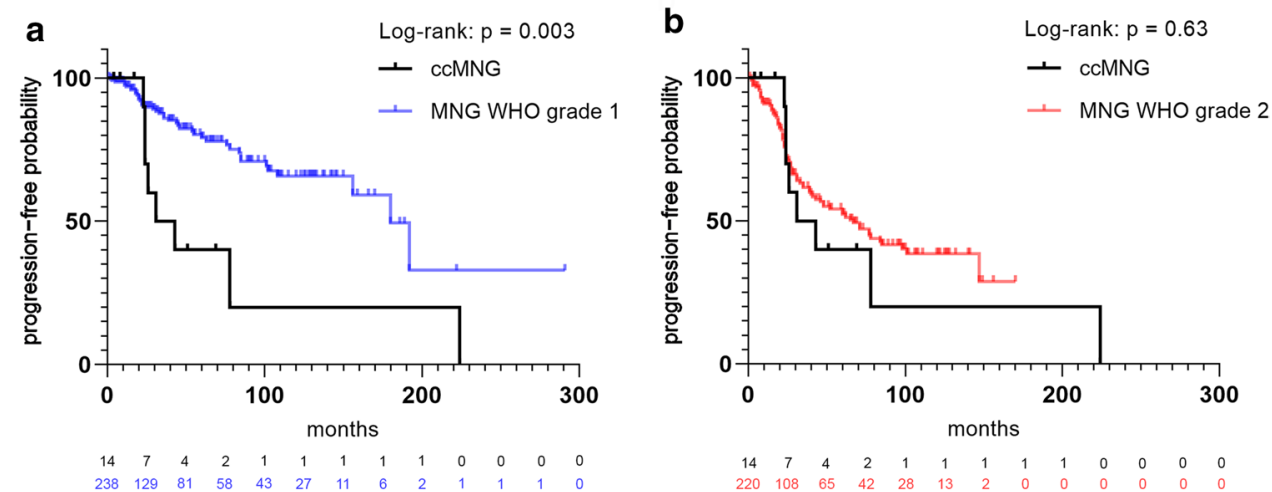


\section{Discussion}

Besides confirming the known association of SMARCE1 mutations and clear cell meningioma, our findings indicate the existence of a highly distinct epigenetic signature of this meningioma subtype. Given previous studies in multiple tumor types on the robustness of cell-of-origin imprints in the epigenome of tumors [2, 6, 7, 25], this may suggest that these tumors arise from a different precursor cell population than the broad spectrum of the other meningioma subtypes. Interestingly, none of the other meningioma subtypes with characteristic alterations, e.g. mutations in $N F 2$, TRAF7/KLF4 or BAP1, or YAP1 fusion, seem to form epigenetic clusters that are distinct to the same extent [19, 20]. However, given that SMARCE1 is itself a broad epigenetic regulator, this could explain why the DNA methylation profile in SMARCE1 deficient meningiomas is so different from that of other meningiomas. However, this finding will need confirmation in subsequent follow-up studies.

Although the clear cell subtype of meningioma represented the most common initial diagnosis in the primarily identified cohort, a smaller number of tumors were initially designated to other histological variants of meningioma (in particular diagnosed as atypical meningioma WHO grade 2). However, a histopathological review for all cases with sufficient material confirmed clearly recognizable cellular areas composed of round to polygonal clear cells with perivascular and interstitial collagen corresponding to the typical histological features of clear cell meningioma in each of the analyzed tumors. On the other hand, none of the histologically diagnosed clear cell meningiomas were found outside this specific cluster, which underlines a strong phenotype/genotype correlation. Therefore, in cases with diagnostic uncertainty immunostaining for SMARCE1 and DNA methylation profiling may be useful for accurate diagnosis of this subtype.

As SMARCE1 mutations were detected in almost all tumors analyzed, it seems that clear cell meningiomas are primarily driven by these alterations. Germline loss-offunction mutations in SMARCE1 were identified in familial spinal meningiomas in 2013 by Smith et al. [22] and seem to be highly linked to the clear cell subtype [23, 26]. SMARCE1 encodes for a subunit of the SWI/SNF complex, which has been shown to play a widespread role in tumorigenesis [13, 15]. Thus, SMARCE1 has been suggested as the potential oncogenic driver in clear cell meningioma [22]. Genetic alterations involving several other subunits of the SWI/SNF complex (in particular SMARCB1 and ARIDIA) are found in meningioma pathogenesis and seem to be associated with more aggressive subtypes of meningioma [3]. In addition, $\mathrm{PRC} 2$, which acts to antagonize the SWI/SNF complex, is upregulated in higher-grade meningiomas [3,5], which is consistent with our results. Inactivating mutations in SWI/ SNF subunits have been found in various other cancers, some of these demonstrating clear cell phenotype of the tumor cells as well $[8,17,27,28]$. Interestingly, SMARCE1 mutations seem to be restricted to this specific epigenetic class of clear cell meningiomas since no SMARCE1 alterations were found in 705 other meningioma samples with available DNA methylation and sequencing data.

In line with our data, clear cell meningioma harboring a SMARCE1 mutation have been commonly described in children and young adults [26]. Interestingly though, the age range in our cohort was relatively wide considering such a molecularly homogeneous tumor, suggesting a possible cell-of-origin, which is present throughout life. Analysis of time to progression or recurrence of these patients in comparison to those with meningioma $\mathrm{WHO}$ grade 2 revealed a similar outcome and hence supports the assignment of WHO grade 2 to these tumors. Whether germline mutations in the SMARCE1 gene are associated with an increased risk for developing multiple meningiomas, as initially suggested [22], has to be determined in subsequent studies.

In summary, our data demonstrate a highly distinct epigenetic signature of clear cell meningiomas, that is associated with frequent mutations within the SMARCE1 gene and/or loss of SMARCE1 protein expression, presumably by other mechanisms. Whether or not this has further implications for tumor classification as a subtype versus an entity requires further study.

Acknowledgements For excellent technical support we sincerely thank the Microarray Unit of the German Cancer Research Center (DKFZ) Genomics and Proteomics Core Facility, as well as L. Dörner, Lea Hoffmann, Moritz Schalles and A. Habel (Department of Neuropathology, Institute of Pathology, University Hospital Heidelberg, Heidelberg, Germany). This study was supported by the Hertie Network of Excellence in Clinical Neuroscience, the German Cancer Aid (70112956), and the Else Kröner-Fresenius Stiftung (EKFS 2015_A60). P. Sievers is a fellow of the Hertie Academy of Excellence in Clinical Neuroscience. F. Sahm is a fellow of the Else Kröner Excellence Program of the Else Kröner-Fresenius Stiftung (EKFS 2017_EKES.24). S. Brandner and Z. Jaunmuktane are supported by the UK Department of Health's NIHR Biomedical Research Centre's funding scheme to UCLH. A proportion of data were obtained from University College London NHS Foundation Trust as part of the UK Brain Archive Information Network (BRAIN UK) which is funded by the Medical Research Council and Brain Tumour Research. BRAIN UK reference number: 19/002.

Funding Open Access funding enabled and organized by Projekt DEAL.

Open Access This article is licensed under a Creative Commons Attribution 4.0 International License, which permits use, sharing, adaptation, distribution and reproduction in any medium or format, as long as you give appropriate credit to the original author(s) and the source, provide a link to the Creative Commons licence, and indicate if changes were made. The images or other third party material in this article are included in the article's Creative Commons licence, unless indicated otherwise in a credit line to the material. If material is not included in the article's Creative Commons licence and your intended use is not 
permitted by statutory regulation or exceeds the permitted use, you will need to obtain permission directly from the copyright holder. To view a copy of this licence, visit http://creativecommons.org/licenses/by/4.0/.

\section{References}

1. Aryee MJ, Jaffe AE, Corrada-Bravo H, Ladd-Acosta C, Feinberg AP, Hansen KD et al (2014) Minfi: a flexible and comprehensive Bioconductor package for the analysis of Infinium DNA methylation microarrays. Bioinformatics 30:1363-1369. https://doi. org/10.1093/bioinformatics/btu049

2. Capper D, Jones DTW, Sill M, Hovestadt V, Schrimpf D, Sturm D et al (2018) DNA methylation-based classification of central nervous system tumours. Nature 555:469-474. https://doi.org/10.1038/ nature26000

3. Collord G, Tarpey P, Kurbatova N, Martincorena I, Moran S, Castro $M$ et al (2018) An integrated genomic analysis of anaplastic meningioma identifies prognostic molecular signatures. Sci Rep 8:13537. https://doi.org/10.1038/s41598-018-31659-0

4. Dobin A, Davis CA, Schlesinger F, Drenkow J, Zaleski C, Jha S et al (2013) STAR: ultrafast universal RNA-seq aligner. Bioinformatics 29:15-21. https://doi.org/10.1093/bioinformatics/bts635

5. Harmanci AS, Youngblood MW, Clark VE, Coskun S, Henegariu O, Duran D et al (2017) Integrated genomic analyses of de novo pathways underlying atypical meningiomas. Nat Commun 8:14433. https://doi.org/10.1038/ncomms14433

6. Hovestadt V, Jones DT, Picelli S, Wang W, Kool M, Northcott PA et al (2014) Decoding the regulatory landscape of medulloblastoma using DNA methylation sequencing. Nature 510:537-541. https://doi.org/10.1038/nature13268

7. Hovestadt V, Remke M, Kool M, Pietsch T, Northcott PA, Fischer $R$ et al (2013) Robust molecular subgrouping and copy-number profiling of medulloblastoma from small amounts of archival tumour material using high-density DNA methylation arrays. Acta Neuropathol 125:913-916. https://doi.org/10.1007/s00401-013-1126-5

8. Jones S, Wang TL, Shih Ie M, Mao TL, Nakayama K, Roden R et al (2010) Frequent mutations of chromatin remodeling gene ARID1A in ovarian clear cell carcinoma. Science 330:228-231. https://doi.org/10.1126/science.1196333

9. Li B, Dewey CN (2011) RSEM: accurate transcript quantification from RNA-Seq data with or without a reference genome. BMC Bioinformatics 12:323. https://doi.org/10.1186/1471-2105-12-323

10. Louis DNOH, Wiestler OD, Cavenee WK (2016) WHO classification of tumours of the central nervous system, vol Revised, 4th edn. IARC, Lyon

11. Love MI, Huber W, Anders S (2014) Moderated estimation of fold change and dispersion for RNA-seq data with DESeq2. Genome Biol 15:550. https://doi.org/10.1186/s13059-014-0550-8

12. Manivel JC, Sung JH (1990) Pathology of meningiomas. Pathol Annu 25:159-192

13. Masliah-Planchon J, Bieche I, Guinebretiere JM, Bourdeaut F, Delattre O (2015) SWI/SNF chromatin remodeling and human malignancies. Annu Rev Pathol 10:145-171. https://doi. org/10.1146/annurev-pathol-012414-040445

14. McPherson A, Hormozdiari F, Zayed A, Giuliany R, Ha G, Sun MG et al (2011) deFuse: an algorithm for gene fusion discovery in tumor RNA-Seq data. PLoS Comput Biol 7:e1001138. https:// doi.org/10.1371/journal.pcbi.1001138

15. Mittal P, Roberts CWM (2020) The SWI/SNF complex in cancer biology, biomarkers and therapy. Nat Rev Clin Oncol 17:435-448. https://doi.org/10.1038/s41571-020-0357-3

16. Ostrom QT, Cioffi G, Gittleman H, Patil N, Waite K, Kruchko C et al (2019) CBTRUS statistical report: primary brain and other central nervous system tumors diagnosed in the United States in 2012-2016. Neuro Oncol 21:v1-v100. https://doi.org/10.1093/ neuonc/noz150

17. Piva F, Santoni M, Matrana MR, Satti S, Giulietti M, Occhipinti G et al (2015) BAP1, PBRM1 and SETD2 in clear-cell renal cell carcinoma: molecular diagnostics and possible targets for personalized therapies. Expert Rev Mol Diagn 15:1201-1210. https:// doi.org/10.1586/14737159.2015.1068122

18. Sahm F, Schrimpf D, Jones DT, Meyer J, Kratz A, Reuss D et al (2016) Next-generation sequencing in routine brain tumor diagnostics enables an integrated diagnosis and identifies actionable targets. Acta Neuropathol 131:903-910. https://doi.org/10.1007/ s00401-015-1519-8

19. Sahm F, Schrimpf D, Stichel D, Jones DTW, Hielscher T, Schefzyk S et al (2017) DNA methylation-based classification and grading system for meningioma: a multicentre, retrospective analysis. Lancet Oncol 18:682-694. https://doi.org/10.1016/ S1470-2045(17)30155-9

20. Sievers P, Chiang J, Schrimpf D, Stichel D, Paramasivam N, Sill $M$ et al (2020) YAP1-fusions in pediatric NF2-wildtype meningioma. Acta Neuropathol 139:215-218. https://doi.org/10.1007/ s00401-019-02095-9

21. Smith MJ, Ahn S, Lee JI, Bulman M, Plessis DD, Suh YL (2017) SMARCE1 mutation screening in classification of clear cell meningiomas. Histopathology 70:814-820. https://doi.org/10.1111/ his. 13135

22. Smith MJ, O'Sullivan J, Bhaskar SS, Hadfield KD, Poke G, Caird $\mathrm{J}$ et al (2013) Loss-of-function mutations in SMARCE1 cause an inherited disorder of multiple spinal meningiomas. Nat Genet 45:295-298. https://doi.org/10.1038/ng.2552

23. Smith MJ, Wallace AJ, Bennett C, Hasselblatt M, Elert-Dobkowska E, Evans LT et al (2014) Germline SMARCE1 mutations predispose to both spinal and cranial clear cell meningiomas. J Pathol 234:436-440. https://doi.org/10.1002/path.4427

24. Stichel D, Schrimpf D, Casalini B, Meyer J, Wefers AK, Sievers P et al (2019) Routine RNA sequencing of formalin-fixed paraffinembedded specimens in neuropathology diagnostics identifies diagnostically and therapeutically relevant gene fusions. Acta Neuropathol 138:827-835. https://doi.org/10.1007/s00401-01902039-3

25. Sturm D, Orr BA, Toprak UH, Hovestadt V, Jones DTW, Capper $D$ et al (2016) New brain tumor entities emerge from molecular classification of CNS-PNETs. Cell 164:1060-1072. https://doi. org/10.1016/j.cell.2016.01.015

26. Tauziede-Espariat A, Parfait B, Besnard A, Lacombe J, Pallud J, Tazi S et al (2018) Loss of SMARCE1 expression is a specific diagnostic marker of clear cell meningioma: a comprehensive immunophenotypical and molecular analysis. Brain Pathol 28:466-474. https://doi.org/10.1111/bpa.12524

27. Wiegand KC, Shah SP, Al-Agha OM, Zhao Y, Tse K, Zeng T et al (2010) ARID1A mutations in endometriosis-associated ovarian carcinomas. N Engl J Med 363:1532-1543. https://doi. org/10.1056/NEJMoa1008433

28. Xia QY, Zhan XM, Fan XS, Ye SB, Shi SS, Li R et al (2017) BRM/SMARCA2-negative clear cell renal cell carcinoma is associated with a high percentage of BRM somatic mutations, deletions and promoter methylation. Histopathology 70:711-721. https://doi.org/10.1111/his.13120

29. Zorludemir S, Scheithauer BW, Hirose T, Van Houten C, Miller G, Meyer FB (1995) Clear cell meningioma. A clinicopathologic study of a potentially aggressive variant of meningioma. Am J Surg Pathol 19:493-505

Publisher's Note Springer Nature remains neutral with regard to jurisdictional claims in published maps and institutional affiliations. 


\section{Affiliations}

Philipp Sievers ${ }^{1,2} \oplus \cdot$ Martin Sill $^{3,4} \cdot$ Christina Blume $^{1,2,5} \cdot$ Arnault Tauziede-Espariat $^{6} \cdot$ Daniel Schrimpf $^{1,2}$.

Damian Stichel ${ }^{1,2}$. David E. Reuss ${ }^{1,2} \cdot$ Helin Dogan $^{1,2}$. Christian Hartmann ${ }^{7}$. Christian Mawrin ${ }^{8}$. Martin Hasselblatt ${ }^{9}$. Walter Stummer ${ }^{10}$. Uta Schick ${ }^{11}$. Jürgen Hench ${ }^{12}$. Stephan Frank ${ }^{12}$. Ralf Ketter ${ }^{13}$. Leonille Schweizer ${ }^{14,15}$. Jens Schittenhelm ${ }^{16}$. Stéphanie Puget ${ }^{17}$. Sebastian Brandner ${ }^{18,19} \cdot$ Zane Jaunmuktane $^{18,20} \cdot$ Benno Küsters $^{21}$. Zied Abdullaev ${ }^{22}$ - Melike Pekmezci ${ }^{23}$. Matija Snuderl ${ }^{24}$ - Miriam Ratliff ${ }^{25} \cdot$ Christel Herold-Mende $^{26}$. Andreas Unterberg $^{27}$ - Kenneth Aldape ${ }^{22}$. David W. Ellison ${ }^{28}$. Pieter Wesseling ${ }^{29,30}$. Guido Reifenberger ${ }^{31,32}$. Wolfgang Wick ${ }^{33,34}$ - Arie Perry ${ }^{23,35}$. Pascale Varlet ${ }^{6} \cdot$ Stefan M. Pfister ${ }^{3,4,36}$ - David T. W. Jones ${ }^{3,37}$. Andreas von Deimling ${ }^{1,2}$. Felix Sahm ${ }^{1,2,3}$ - The German Consortium “Aggressive Meningiomas"

1 Department of Neuropathology, Institute of Pathology, University Hospital Heidelberg, Heidelberg, Germany

2 Clinical Cooperation Unit Neuropathology, German Consortium for Translational Cancer Research (DKTK), German Cancer Research Center (DKFZ), Heidelberg, Germany

3 Hopp Children's Cancer Center Heidelberg (KiTZ), Heidelberg, Germany

4 Division of Pediatric Neurooncology, German Cancer Consortium (DKTK), German Cancer Research Center (DKFZ), Heidelberg, Germany

5 Bioinformatics and Omics Data Analytics, German Cancer Research Center (DKFZ), Heidelberg, Germany

6 Department of Neuropathology, GHU Paris Psychiatry and Neurosciences, Sainte-Anne Hospital, Paris, France

7 Department of Neuropathology, Institute of Pathology, Hannover Medical School (MHH), Hannover, Germany

8 Department of Neuropathology, Otto-Von-Guericke University, Magdeburg, Germany

9 Institute of Neuropathology, University Hospital Münster, Münster, Germany

10 Department of Neurosurgery, University Hospital Münster, Münster, Germany

11 Department of Neurosurgery, Clemenshospital Münster, Münster, Germany

12 Institute for Medical Genetics and Pathology, University Hospital Basel, Basel, Switzerland

13 Department of Neurosurgery, University Hospital Homburg Saar, Homburg, Germany

14 Department of Neuropathology, Charité-Universitätsmedizin Berlin, Corporate Member of Freie Universität Berlin, Humboldt-Universität zu Berlin, and Berlin Institute of Health, Berlin, Germany

15 German Cancer Consortium (DKTK), Partner Site Berlin, German Cancer Research Center (DKFZ), Heidelberg, Germany

16 Department of Neuropathology, University of Tübingen, Tübingen, Germany
17 Department of Pediatric Neurosurgery, Hôpital Necker-Enfants Malades, APHP, Université de Paris, Paris, France

18 Division of Neuropathology, National Hospital for Neurology and Neurosurgery, University College London Hospitals NHS Foundation Trust, Queen Square, London, UK

19 Department of Neurodegenerative Disease, UCL Queen Square Institute of Neurology, Queen Square, London, UK

20 Department of Clinical and Movement Neurosciences and Queen Square Brain Bank for Neurological Disorders, Queen Square Institute of Neurology, University College London, London, UK

21 Department of Pathology, Radboud University Medical Center, Nijmegen, the Netherlands

22 Laboratory of Pathology, Center for Cancer Research, National Cancer Institute, National Institutes of Health, Bethesda, MD, USA

23 Department of Pathology, University of California, San Francisco, CA, USA

24 Department of Pathology, NYU Langone Medical Center, New York, NY, USA

25 Department of Neurosurgery, University Medical Centre Mannheim, University of Heidelberg, Mannheim, Germany

26 Division of Experimental Neurosurgery, Department of Neurosurgery, University Hospital Heidelberg, Heidelberg, Germany

27 Department of Neurosurgery, University Hospital Heidelberg, Heidelberg, Germany

28 Department of Pathology, St. Jude Children's Research Hospital, Memphis, TN, USA

29 Department of Pathology, Amsterdam University Medical Centers, Location VUmc and Brain Tumor Center Amsterdam, Amsterdam, The Netherlands

30 Princess Máxima Center for Pediatric Oncology, Utrecht, The Netherlands

31 Institute of Neuropathology, Heinrich Heine University, Düsseldorf, Germany

32 German Cancer Consortium (DKTK), Partner Site Essen/Düsseldorf, Germany 
33 Clinical Cooperation Unit Neurooncology, German Consortium for Translational Cancer Research (DKTK), German Cancer Research Center (DKFZ), Heidelberg, Germany

34 Department of Neurology and Neurooncology Program, National Center for Tumor Diseases, Heidelberg University Hospital, Heidelberg, Germany

35

Department of Neurological Surgery, University of California, San Francisco, CA, USA
36 Department of Pediatric Oncology, Hematology, Immunology and Pulmonology, University Hospital Heidelberg, Heidelberg, Germany

37 Pediatric Glioma Research Group, German Cancer Research Center (DKFZ), Heidelberg, Germany 